\title{
Design, performance, and calibration of CMS hadron-barrel calorimeter wedges
}

The CMS-HCAL Collaboration

S. Abdullin ${ }^{23,20}$, V. Abramov ${ }^{12}$, B. Acharya ${ }^{7}$, M. Adams ${ }^{22}$, N. Akchurin ${ }^{26, a}$, U. Akgun ${ }^{25}$, E.W. Anderson ${ }^{19}$, G. Antchev ${ }^{21}$, S. Ayann ${ }^{25}$, S. Aydin ${ }^{14}$, M. Baarmand ${ }^{27}$, D. Baden ${ }^{23}$, Sud. Banerjee ${ }^{7}$, Sun. Banerjee, R. Bard ${ }^{23}$, V. Barnes ${ }^{34}$, H. Bawa ${ }^{6}$, G. Baiatian ${ }^{1}$, G. Bencze ${ }^{5}$, S. Beri ${ }^{6}$, V. Bhatnagar ${ }^{6}$, A. Bodek ${ }^{32}$, H. Budd ${ }^{32}$, K. Burchesky ${ }^{22}$, T. Camporesi ${ }^{13}$, K. Cankoçak ${ }^{15,35}$, K. Carrell ${ }^{26}$, S. Chendvankar ${ }^{7}$, Y. Chung ${ }^{32}$, L. Cremaldi ${ }^{29}$, P. Cushman ${ }^{28}$,

J. Damgov ${ }^{3}$, P. de Barbaro ${ }^{32}$, A. Demianov ${ }^{11}$, T. de Visser ${ }^{13}$, L. Dimitrov ${ }^{3}$, S. Dugad ${ }^{7}$, I. Dumanoglu ${ }^{14}$, F. Duru ${ }^{25}$,

J. Elias $^{20}$, D. Elvira ${ }^{20}$, I. Emeliantchik ${ }^{2}$, S. Eno ${ }^{23}$, A. Ershov ${ }^{11}$, E. Eskut ${ }^{14}$, W. Fisher ${ }^{31}$, J. Freeman ${ }^{20}$,

V. Gavrilov ${ }^{10}$, V. Genchev ${ }^{3}$, Y. Gershtein ${ }^{10,33}$, I. Golutvin ${ }^{9}$, P. Goncharov ${ }^{12}$, T. Grassi ${ }^{23}$, D. Green ${ }^{20}$,

A. Gribushin ${ }^{11}$, B. Grinev ${ }^{18}$, E. Gülmez ${ }^{16}$, K. Gümüsş ${ }^{2}$, T. Haelen ${ }^{32}$, S. Hagopian ${ }^{33}$, V. Hagopian ${ }^{33}$, J. Hauptman ${ }^{19}$, E. Hazen ${ }^{21}$, A. Heering ${ }^{28,21}$, M. Imboden ${ }^{32,21}$, E. Isiksal ${ }^{16,36}$, C. Jarvis ${ }^{23}$, K. Johnson ${ }^{33}$, V. Kaftanov ${ }^{10, \dagger}$,

V. Kalagin ${ }^{9}$, D. Karmgard ${ }^{30}$, S. Kalmani ${ }^{7}$, S. Katta ${ }^{7}$, M. Kaur ${ }^{6}$, M. Kaya ${ }^{16,37}$, A. Kayis-Topaksu ${ }^{14}$, R. Kellogg ${ }^{23}$,

A. Khmelnikov ${ }^{12}$, I. Kisselevich ${ }^{10}$, O. Kodolova ${ }^{11}$, J. Kohli ${ }^{6}$, V. Kolossov ${ }^{10}$, A. Korablev ${ }^{12}$, Y. Korneev ${ }^{12}$,

I. Kosarev ${ }^{9}$, A. Krinitsyn ${ }^{12}$, A. Krokhotin ${ }^{10}$, V. Kryshkin ${ }^{12}$, S. Kuleshov ${ }^{10}$, A. Kumar ${ }^{6}$, S. Kunori ${ }^{23}$, A. Polatoz ${ }^{14}$,

A. Laasanen ${ }^{34}$, C. Lawlor ${ }^{21}$, D. Lazic ${ }^{20,21}$, L. Levchuk ${ }^{17}$, D. Litvintsev ${ }^{10,20}$, L. Litov ${ }^{4}$, S. Los ${ }^{20}$, V. Lubinsky ${ }^{18}$,

V. Lukanin ${ }^{12}$, E. Machado ${ }^{21}$, J. Mans ${ }^{31,28}$, V. Massolov ${ }^{2}$, K. Mazumdar ${ }^{7}$, J.P. Merlo ${ }^{25}$, G. Mescheryakov ${ }^{9}$,

A. Mestvirishvili ${ }^{25}$, M. Miller ${ }^{25}$, N. Mondal ${ }^{7}$, P. Nagaraj ${ }^{7}$, E. Norbeck ${ }^{25}$, V. O'Dell ${ }^{20}$, J. Olson ${ }^{25}$, Y. Onel ${ }^{25}$,

G. Onengut ${ }^{14}$, N. Ozdes-Koca ${ }^{14}$, S. Ozkorucuklu ${ }^{16,38}$, F. Ozok ${ }^{15}$, S. Paktinat ${ }^{8,39}$, M. Patil ${ }^{7}$, S. Petrushanko ${ }^{11}$,

V. Pikalov ${ }^{12}$, S. Piperov ${ }^{3}$, V. Podrasky ${ }^{24}$, A. Pomposs ${ }^{34}$ C. Posch ${ }^{21}$, W. Qian ${ }^{22}$, R. Ralich ${ }^{27}$, L. Reddy ${ }^{7}$, J. Reidy ${ }^{29}$,

R. Ruchti ${ }^{30}$, J. Rohlf ${ }^{21}$, A. Ronzhinn ${ }^{20}$, A. Ryazanov ${ }^{12}$, D.A. Sanders ${ }^{29}$, C. Sanzeni ${ }^{24}$, L. Sarycheva ${ }^{11}$,

B. Satyanarayana ${ }^{7}$, I. Schmidt ${ }^{25}$, V. Senchishin ${ }^{18}$, S. Sergeyev ${ }^{20}$, M. Serin-Zeyrek ${ }^{15}$, R. Sever ${ }^{15}$, J. Singh ${ }^{6}$,

A. Sirunyan ${ }^{1}$, A. Skuja ${ }^{23}$, B. Sherwood ${ }^{28}$, N. Shumeiko ${ }^{2}$, V. Smirnov ${ }^{9}$, P. Sorokin ${ }^{17}$, R. Stefanovich ${ }^{2}$, V. Stolin ${ }^{10}$,

K. Sudhakar ${ }^{7}$, I. Suzuki ${ }^{20}$, V. Talov ${ }^{12}$, R. Thomas $^{26}$, C. Tully ${ }^{31}$, L. Turchanovich ${ }^{12}$, A. Ulyanov ${ }^{10}$, I. Vankov ${ }^{3}$,

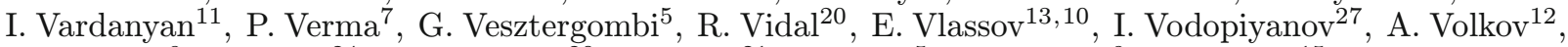

A. Volodko ${ }^{9}$, D. Winn ${ }^{24}$, J. Whitmore ${ }^{20}$, S.X. Wu ${ }^{21}$, P. Zalan ${ }^{5}$, A. Zarubin ${ }^{9}$, M. Zeyrek $^{15}$

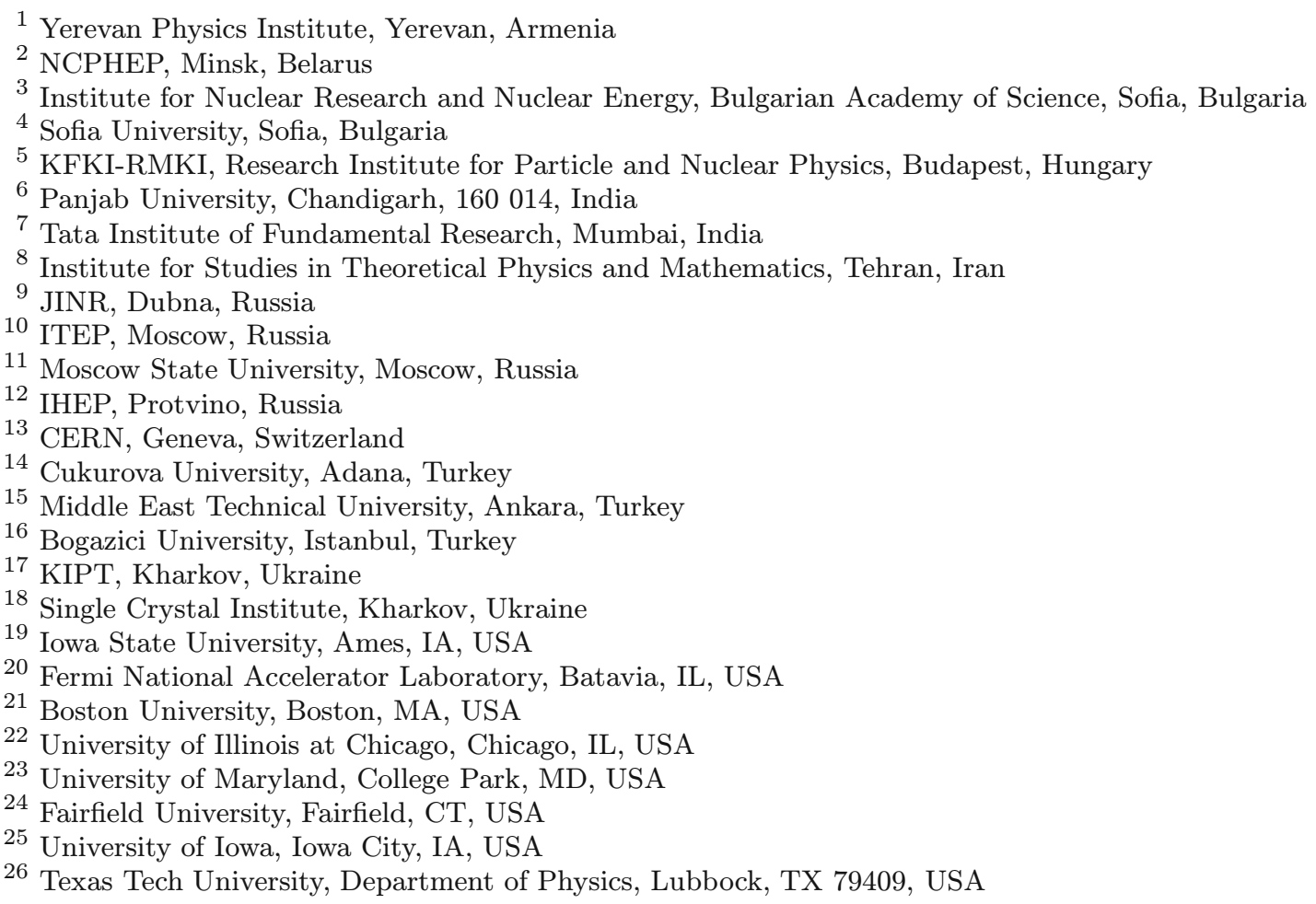


27 Florida Institute of Technology, Melbourne, FL, USA

28 University of Minnesota, Minneapolis, MN, USA

29 University of Mississippi, Oxford, MS, USA

30 University of Notre Dame, Notre Dame, IN, USA

31 Princeton University, Princeton, NJ, USA

32 University of Rochester, Rochester, NY, USA

33 Florida State University, Tallahassee, FL, USA

34 Purdue University, West Lafayette, IN, USA

35 Now at: Mugla University, Mugla, Turkey

36 Now at: Marmara University, Istanbul, Turkey

37 Now at: Kafkas University, Kars, Turkey

38 Now at: Suleyman Demirel University, Isparta, Turkey

39 Now at: Sharif University of Technology, Tehran, Iran

$\dagger$ deceased

Received: 13 June 2007 / Revised version: 25 February 2008 /

Published online: 9 April 2008 - @ S Springer-Verlag / Società Italiana di Fisica 2008

\begin{abstract}
Extensive measurements have been made with pions, electrons and muons on four production wedges of the compact muon solenoid (CMS) hadron barrel (HB) calorimeter in the H2 beam line at CERN with particle momenta varying from 20 to $300 \mathrm{GeV} / c$. The time structure of the events was measured with the full chain of preproduction front-end electronics running at $34 \mathrm{MHz}$. Moving-wire radioactive source data were also collected for all scintillator layers in the HB. The energy dependent time slewing effect was measured and tuned for optimal performance.
\end{abstract}

\section{Introduction}

The Compact Muon Solenoid (CMS) is a general purpose experiment designed to study $p p$ collisions at the Large Hadron Collider (LHC) [1,2]. The CMS detector calorimeter has been designed to detect cleanly the diverse signatures of new physics through the measurement of jets with moderate precision and by measuring missing transverse energy flow. The design goal of the calorimeter is to have an energy resolution of $100 \% / \sqrt{E} \oplus 5 \%$ where $E$ is in $\mathrm{GeV}$ [3]. The CMS experiment has a $4 \mathrm{~T}$ superconducting solenoidal magnet of length $13 \mathrm{~m}$ and inner diameter $5.9 \mathrm{~m}$. The magnet determines many of the features of the CMS calorimeters because the barrel and end-cap calorimeters are located inside this magnet. The hadron calorimeter (HCAL) plays a fundamental role in most anticipated discoveries at the LHC. The HCAL is used to measure the timing and energy of hadronic showers, as well as their angle and position, needed for the generation of level-1 trigger primitives, the high level trigger, and offline reconstruction of jets and missing transverse energy [4-6]. Figure 1 illustrates the calorimeters in and around the CMS solenoidal magnet. The cylindrically symmetric hadron barrel (HB) calorimeter consisting of alternating layers of brass and plastic scintillator plates surrounds the lead tungstate $\left(\mathrm{PbWO}_{4}\right)$ electromagnetic calorimeter (ECAL$\mathrm{EB})$. The HB design maximizes the number of interaction lengths inside the coil, which requires minimizing the amount of space devoted to the active medium. The scintillator tiles are read out with embedded wavelength shifting (WLS) fibers which achieves the required compact and

\footnotetext{
a e-mail: Nural.Akchurin@ttu.edu
}

hermetic design. This technology was first developed at Protvino and by the UA1 collaboration and has been successfully used in an upgrade of the CDF endcap calorimeter $[7-10]$. Brass was chosen as the absorber material because it is non-magnetic. This design makes construction relatively simple, lends itself to projective tower geometry, and eliminates uninstrumented gaps.

The CMS HCAL contains 9072 readout channels organized into four subsystems: barrel (HB, 2592 channels), endcap (HE, 2592 channels), outer (HO, 2160 channels) and forward (HF, 1728 channels). This paper addresses the design, performance, and calibration of the HB. The performance of the $\mathrm{HE}, \mathrm{HO}$, and $\mathrm{HF}$ were also extensively investigated and are reported elsewhere [11-14].

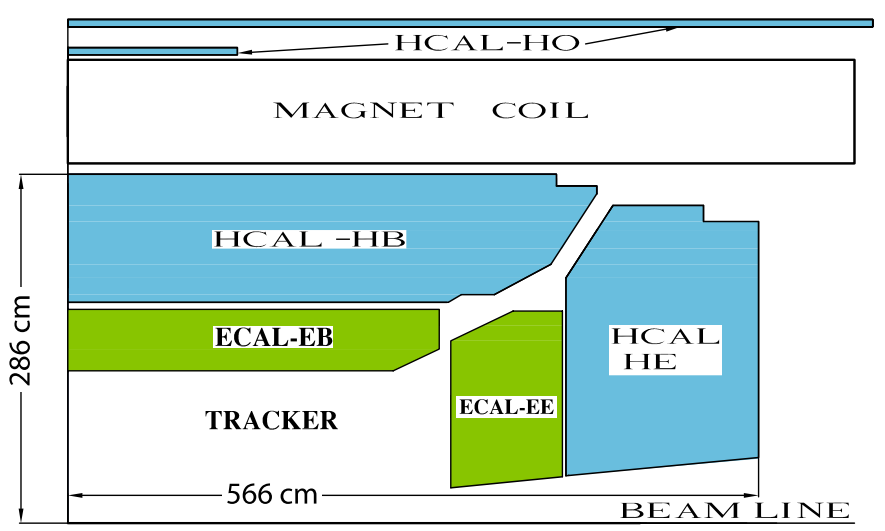

Fig. 1. Location of the ECAL and the HCAL detectors (quarter slice-longitudinal cross section) in and around the CMS magnet 
While the beam tests of the HB prototype and bench tests of the calibration systems were reported previously $[15,16]$, this paper reports the first measurements of production modules with the complete electronics chain. As discussed in Sect. 6, one of the goals of this study was to relate the radioactive source measurements to beam measurements in order to calibrate the HB wedges which were not exposed to particle beams. After these measurements were performed, the $\mathrm{HB}$ wedges were assembled into the final barrel configuration.

This paper is organized as follows. The design details of the hadronic calorimeter are presented in Sect. 2. Topics related to electronics and data acquisition and the test beam setup are in Sects. 3 and 4. Section 5 gives the $\mathrm{HB}$ performance in particle beams. Following a summary of the radioactive source calibration in Sect. 6, we draw conclusions and summarize our work in Sect. 7.

\section{HCAL barrel design}

\subsection{Absorber geometry}

The HB covers the pseudorapidity range $-1.3<\eta<1.3$ and consists of 36 identical azimuthal wedges $\left(\Delta \phi=20^{\circ}\right)$ which form two half-barrels ( $\mathrm{HB}+$ and $\mathrm{HB}-$ ). Each halfbarrel is inserted from either end of the cryostat of the superconducting solenoid. Each wedge is further segmented into four azimuthal $\left(\Delta \phi=5^{\circ}\right)$ sectors. The plates are bolted together in a staggered geometry resulting in a configuration that contains no projective passive material for the full radial extent of a wedge (see Fig. 2). The innermost and outermost plates are made of stainless steel to provide structural strength. The scintillator

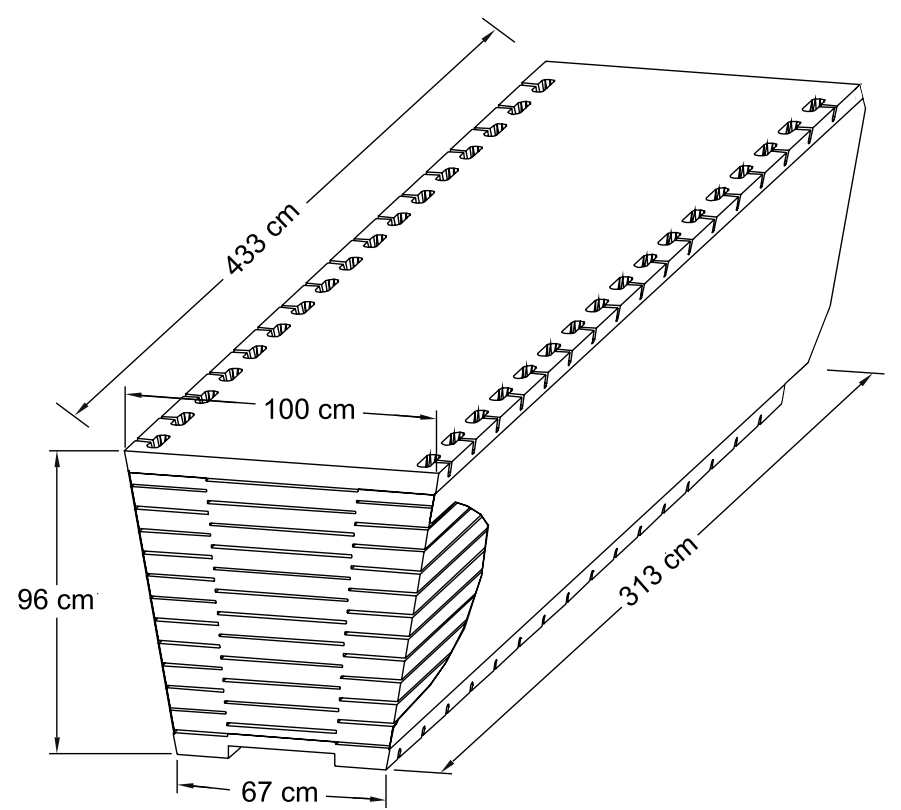

Fig. 2. Isometric view of an $\mathrm{HB}$ wedge: the scintillator trays (Fig. 3) are inserted into slots at the end of the wedge is divided into $16 \eta$ sectors, resulting in a segmentation of $(\Delta \eta, \Delta \phi)=(0.087,0.087)$. The wedges are bolted together and the gap between the wedges is less than $2 \mathrm{~mm}$.

The absorber itself consists of a $40 \mathrm{~mm}$ thick front steel plate, followed first by eight $50.5 \mathrm{~mm}$ thick brass plates, and then six $56.5 \mathrm{~mm}$ thick brass plates, with a final $75 \mathrm{~mm}$ thick steel back plate. The total absorber thickness at $90^{\circ}$ is 5.82 interaction lengths $\left(\lambda_{\mathrm{I}}\right)$. The HB effective thickness increases with polar angle and is 10.6 $\lambda_{\text {I }}$ at $|\eta|=1.3$. The electromagnetic crystal calorimeter [17] in front of the $\mathrm{HB}$ adds $\sim 1.1 \lambda_{\mathrm{I}}$ independent of $\eta$.

The brass absorber is commonly known as $\mathrm{C} 26000$ (cartridge brass) and composed of $70 \% \mathrm{Cu}$ and $30 \% \mathrm{Zn}$. The density is $8.83 \mathrm{~g} / \mathrm{cm}^{3}$. The radiation length is $X_{0}=1.49 \mathrm{~cm}$ and the nuclear interaction length is $\lambda_{\mathrm{I}}=16.42 \mathrm{~cm}$.

\subsection{Scintillator}

The CMS HCAL active elements consist of about 70000 scintillator tiles. In order to limit the number of individual physical elements, the tiles of a given azimuthal section and depth layer are grouped into a single scintillator unit, referred to as a tray.

Figure 3 shows the end portion of a typical tray. This design proved to be robust and practical. We tested each scintillator tray and the optical readout chain before installation into the absorber structure. The construction of the absorber structure and the scintillator assemblies were independent.

The first layer of scintillator (layer-0) is located in front of the steel support plate and is made of $9-\mathrm{mm}$ thick Bicron BC408. The last scintillator layer (layer-16) is 9-mm thick Kuraray SCSN81. The others are all 3.7-mm thick Kuraray SCSN81 plates. The active material choice for the HB was Kuraray SCSN81 scintillator because of its long-term stability and acceptable radiation hardness.

A tray is made of individual optically independent scintillators with white painted edges wrapped in Tyvek 1073D sheets. The scintillators are attached to a $0.5-\mathrm{mm}$ thick plastic substrate with plastic rivets. Light from each tile

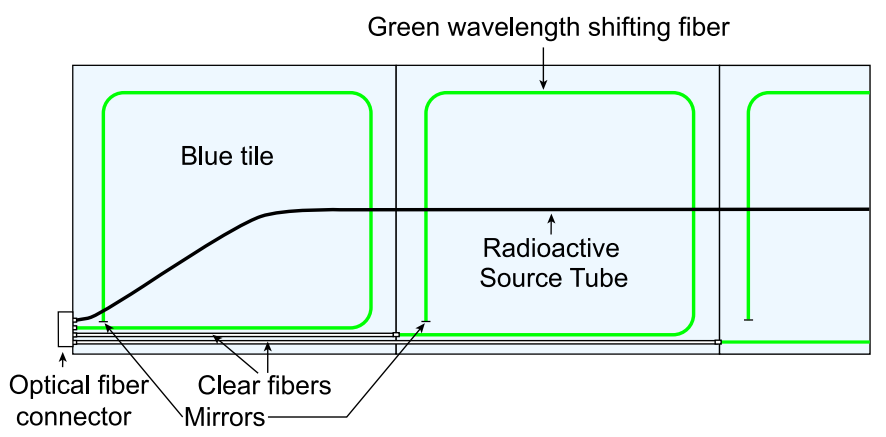

Fig. 3. Schematic of a partial scintillator tray, showing green wavelength shifting fibers, clear fibers, and the radioactive source tube locations 
is collected with a $0.94-\mathrm{mm}$ diameter green double-clad wavelength-shifting fiber (Kuraray Y11) which is inserted in a ball-groove machined in the scintillator. The top of the tray is covered with a $2-\mathrm{mm}$ thick polystyrene sheet which carries the optical readout fibers. The end tips of the wavelength shifting fibers embeded inside the scintillator trays are coated by vacuum evaporation technique with a thin layer of aluminum at the tip which serves as a reflector. The average reflectivity is $\sim 83 \%$ with a spread of about $6.5 \%[18]$.

The wavelength shifting fibers are spliced to clear fibers to minimize optical attenuation in transporting the light from the scintillator plates to the photosensors located a few meters away. A fusion splicer was developed for this task and the light transmission across the splice is $92.6 \%$ with an rms of $1.8 \%$. The clear fiber is terminated at a diamond-finished optical connector at the edge of the scintillator tray. An optical cable then further transports light to an optical unit which arranges the fibers into readout towers and guides the light to a hybrid photodiode (HPD) [19].

The completed tray was tested with a collimated ${ }^{137} \mathrm{Cs}$ source. This source illuminates a 4 -cm diameter spot on the tray and is positioned with a computer controlled translation stage at selected locations on the tray. We determined the relative light yield of each tile and the uniformity of each tray. The light yield of individual tiles has an rms variation of $4.6 \%$, while the transverse uniformity of a tile has a spread of $4.5 \%$. A uniformly illuminated tile has a light yield variation of about $6 \%$. The overall uniformity from tile to tile as measured for a hadron shower is about $8 \%$ per tile. For hadronic showers, the weighted average rms over a tower with 16 tiles is about $3.5 \%$ and for muons it is $8 \% \sqrt{16}=2 \%$.

For calibration purposes, the top of each tray also houses a 1-mm diameter stainless steel tube, called a radioactive source tube, that guides a ${ }^{137} \mathrm{Cs}$ ( or ${ }^{60} \mathrm{Co}$ ) source welded on the tip of a thin stainless steel wire across the center of each tile in a tray (Fig. 3). During the assembly stage, the scintillator trays are tested by guiding the wire source through the source tubes. The rms of the ratio of the light yields with the collimated source to that of the wire source is $1.3 \%$.

There is considerable material between the active elements of the ECAL-EB and HCAL-HB. Originally, layer-0 was planned to be read independently from the other scintillators to sample early hadronic showers initiated either in the EB or in the inert material. Since the original plan was to read layer-0 independently, a thicker $(9 \mathrm{~mm})$ and a brighter scintillator (BC408) was used. It produced about three times more light than the other scintillator layers. For space and other limitations, it was not possible to read layer-0 separately. Since layer-0 was brighter, the optical signals were attenuated by neutral density filters placed between the optical fibers and the HPD housing and added to the light of the other scintillators in the same tower. Transmission filters of 20,30 and $40 \%$ were tested in the beam and the energy resolution had a very broad minimum at $30 \%$ and changed by only $1 \%$ between 20 and $40 \%$. The $30 \%$ transmission neutral density filter was

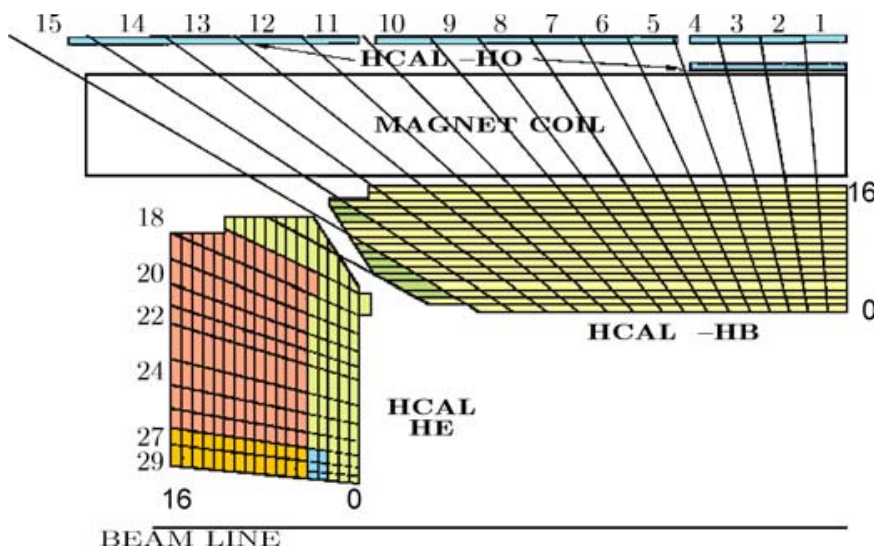

Fig. 4. The HCAL tower segmentation for one-fourth of the $\mathrm{HB}, \mathrm{HO}$, and HE detectors is shown above. The numbers on top and on the left refer to the tower numbers. The numbers on the right and on the bottom (0-16) indicate the scintillator layers numbers inserted into slots in the absorber. The shading represents independent longitudinal readouts in the $\mathrm{HB} / \mathrm{HE}$ overlap and the small angle regions

chosen so the light level is roughly the same as the other 15 layers.

\subsection{Segmentation}

The segmentation of the HB is illustrated in Fig. 4 (Table 1). Towers 1 through 14 all have a single longitudinal readout. Towers 15 and 16 are segmented in depth. The front segment of tower 15 contains either 12 or 13 scintillator layers. The rear segment of Tower 15 has three scintillator layers. Tower 16 has five layers in the front

Table 1. The HB tower sizes and depths are summarized below. The thicknesses refer to the center of the tower. Note that tower 16 overlaps with the HCAL endcap (HE) calorimeter. See also Fig. 4

\begin{tabular}{lcc}
\hline Tower number & $\eta_{\min }-\eta_{\max }$ & Thickness $\left(\lambda_{\mathrm{I}}\right)$ \\
\hline 1 & $0.000-0.087$ & 5.39 \\
2 & $0.087-0.174$ & 5.43 \\
3 & $0.174-0.261$ & 5.51 \\
4 & $0.261-0.348$ & 5.63 \\
5 & $0.348-0.435$ & 5.80 \\
6 & $0.435-0.522$ & 6.01 \\
7 & $0.522-0.609$ & 6.26 \\
8 & $0.609-0.696$ & 6.57 \\
9 & $0.696-0.783$ & 6.92 \\
10 & $0.783-0.870$ & 7.32 \\
11 & $0.870-0.957$ & 7.79 \\
12 & $0.957-1.044$ & 8.30 \\
13 & $1.044-1.131$ & 8.89 \\
14 & $1.131-1.218$ & 9.54 \\
15 & $1.218-1.305$ & 10.3 \\
16 & $1.305-1.392$ & overlaps HE \\
\hline
\end{tabular}




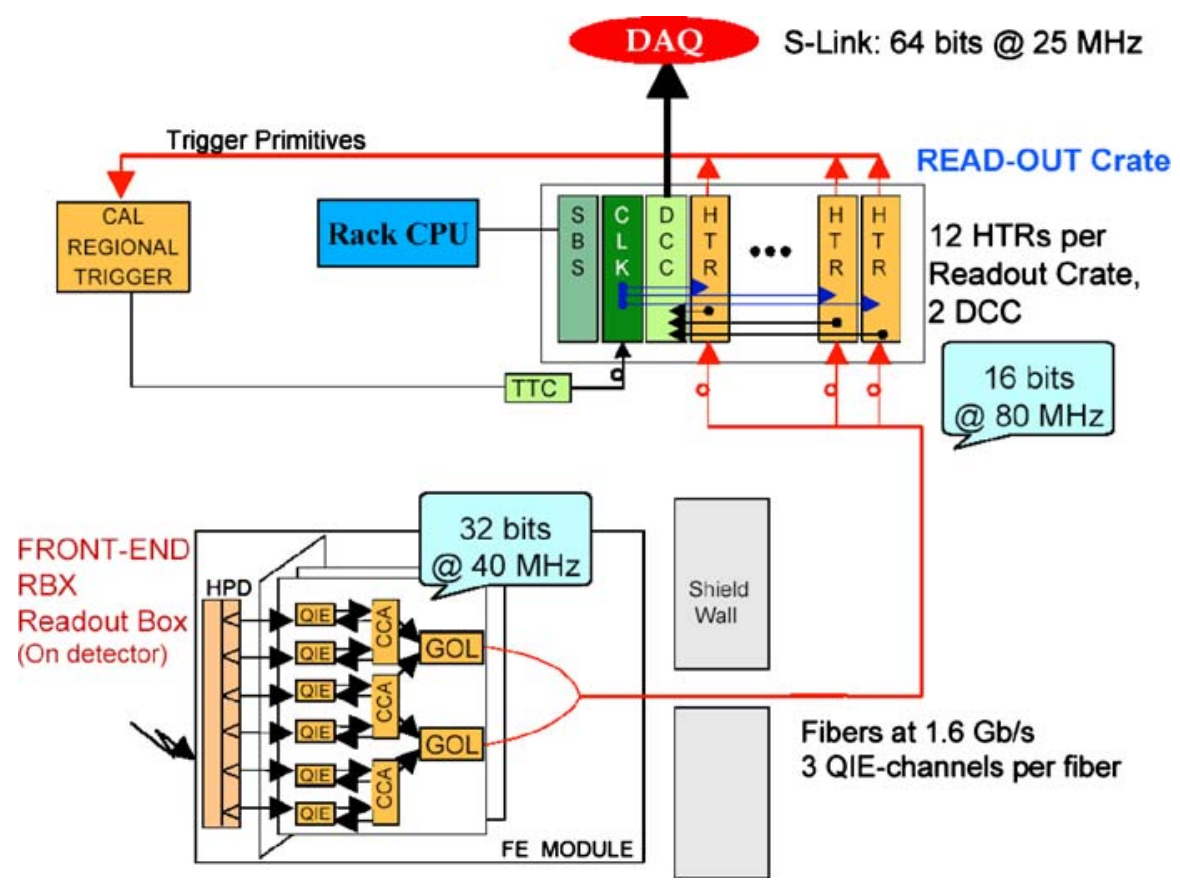

Fig. 5. Overview of the HCAL data acquisition electronics. The data from the HPD are digitized in the QIE and driven off detector by the GOL (Gigabit Optical Link) driving optical fibers. Data are received in the HTR which creates trigger primitives while the data are sent through the DCC to the CMS data acquisition segment and three in the rear. Tower 16 does not have a layer-0 scintillator.

\section{Electronics and data acquisition}

Figure 5 shows an overview of the HCAL electronics and data acquisition system. All key elements were tested during these measurements.

\subsection{Front-end electronics}

Each wedge contains 72 channels of front-end electronics mounted on the detector periphery near tower 14. These circuits are housed in an enclosure referred to as a readout box (RBX). Each of these RBXs is further divided into four readout modules (RM). A single RM contains a 19-channel HPD which registers signals from sixteen independent $(\Delta \eta, \Delta \phi)=(0.087,0.087)$ towers at a fixed $\phi$ angle. The $\mathrm{HPD}^{1}$ is a planar structure consisting of a photocathode and a silicon diode separated by $3.5 \mathrm{~mm}$ of vacuum $[19,20]$. Photoelectrons are accelerated to $\sim 8 \mathrm{kV}$ kinetic energy and strike the diode causing ionization. Collection of the liberated holes leads to a gain of about 1600 . The gain is determined by the accelerating voltage and the value was chosen based on life-time tests. The diode consists of 19 electrically independent readouts. The HPD is a low noise, moderate gain single stage phototube. The major advantage is that it functions inside a magnetic field without shielding, as long as the axis of the tube is aligned with the $B$ field direction. Indeed in the absence of a mag-

1 HPDs are manufactured by DEP, B.V. Delft Electronische Producten, Roden, Netherlands. netic field there is pixel-to-pixel crosstalk in the HPDs of about $8 \%$. However, that is not the case during CMS operation where the magnetic field spirals the electrons in a very tight circle. In addition, the readout channels are arranged such that this $8 \%$ effect is made negligible by summing towers associated with nearest neighbor pixels.

The HPD signals are fed into three 6-channel readout cards located inside the RM. These readout cards are based on a custom ASIC which performs charge integration and encoding (QIE) [21,22]. The QIE is a non-linear multi-range ADC designed to provide approximately constant relative precision over a wide dynamic range of $1 \mathrm{fC}$ to $10000 \mathrm{fC}$. This is accomplished with a floating-point analog-to-digital conversion in which the bin width in each of four ranges is increased in proportion to the input amplitude. The output of the QIE contains 2 bits of range (exponent) and 5 bits of mantissa. In addition, the QIE has four time-interleaved stages.

\subsection{Trigger and readout modules}

The data are sent from the front-end electronics on the detector to HCAL trigger and readout modules (HTR) via gigabit optical links (GOL) housed away from the detector. Each link carries three channels of data. The HTR modules used for these measurements were 24 channel units in a $9 \mathrm{U}$ VME format. A total of six HTR modules were used to read out two HB wedges simultaneously (144 channels).

A block diagram of the HTR is shown in Fig. 6. The HTR is equipped with optical receivers, timing and trigger (TTC) signal circuitry, serial low voltage digital signal (LVDS-Channel Link) outputs to the data concentrator card (DCC), and FPGA for trigger outputs. The optical inputs receive data from the front-end electronics, with one charge sample per LHC bunch crossing. 


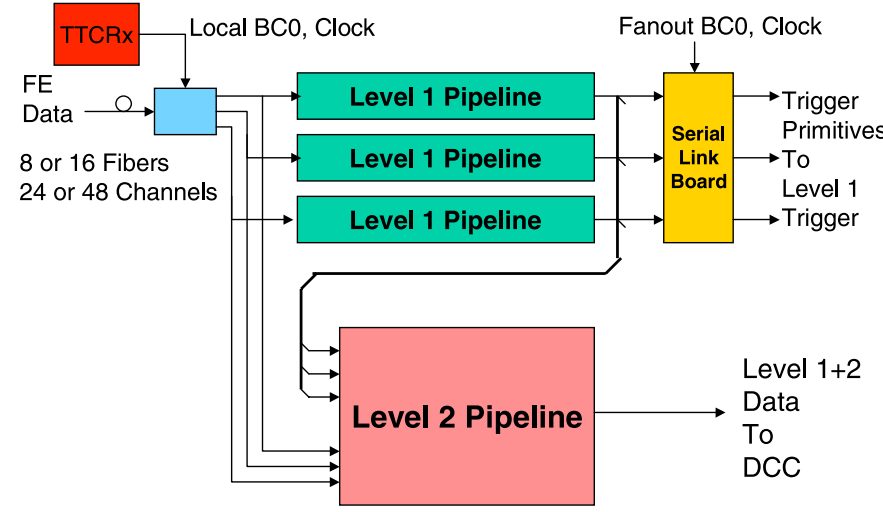

Fig. 6. Block diagram of the HTR electronics. The data from the detector drives two pipelines; one to the CMS trigger and a second to the CMS data acquisition via DCC

The HTR includes two data pipe-lines. The trigger pipe-line assigns the front-end data to a particular LHC bunch crossing and sends them to the CMS trigger. The data acquisition pipe-line stacks the front-end data and sends it to the DCC which performs the data acquisition task.

The raw input data stream in the HTR is deserialized and synchronized to the local clock. A programmable delay of up to a few clock cycles is used to align data from different input fibers. The channel numbers carried on one fiber are demultiplexed. Each channel is then fed to a linearizing look-up table which converts the raw input data to a 16-bit linear energy value. Next, a finite-impulse response (FIR) filter is used to subtract the pedestal and assign all the energy to a single bunch crossing. This performs the same function as a traditional analog shaper, but has the advantage of being easily reprogrammable. Finally, the energy is converted to $E_{\mathrm{T}}$ and compressed to 8 bits according to a non-linear transformation specified by the CMS level- 1 calorimeter trigger, and a comparison is performed to check if the signal may repre- sent a muon. This compressed output plus a muon ID bit is sent to level-1. The final synchronization and serial transmission is performed by a synchronization and link board (SLB).

\subsection{Data concentrator card}

The LVDS link receiver boards use Channel Link [23] technology from National Semiconductor. Each board contains three independent link receivers which can operate at $20-66 \mathrm{MHz}$ (16-bit words). Buffering for $128 \mathrm{~K} \mathrm{32-bit}$ words is provided for each link with provision to discard data if buffer occupancy exceeds a programmable threshold. Event building, protocol checking, event number checking and bit error correction are performed independently for each link.

The DCC logic is designed to operate continuously at the full speed of the two input PCI busses, namely $(33 \mathrm{MHz}) \times(32 \mathrm{bits}) \times(2)$. The event builder and output logic circuitry must thus run at an average rate of at least $66 \mathrm{MHz}$ (32-bit words) or $264 \mathrm{MBytes} / \mathrm{s}$. The event builder output is sent in parallel to several destinations.

The outputs are:

1. The data acquisition (DAQ) output: Every event is sent via SLINK-64 to the CMS DAQ. The contents of each event may be controlled by configuration registers.

2. The trigger data output: The trigger information sent to the CMS level-1 trigger is also sent via SLINK-64 to a special trigger DAQ system for monitoring of the trigger performance.

3. The spy output: A selected subset of events is sent to a VME-accessible memory for monitoring and diagnostics.

Error detection and recovery are a primary consideration in a large synchronous system and the DCC contains logic dedicated to this purpose. Figure 7 shows the main DAQ data pipe-line and buffering. Hamming error correction is used for the LVDS links between the HTR and DCC.

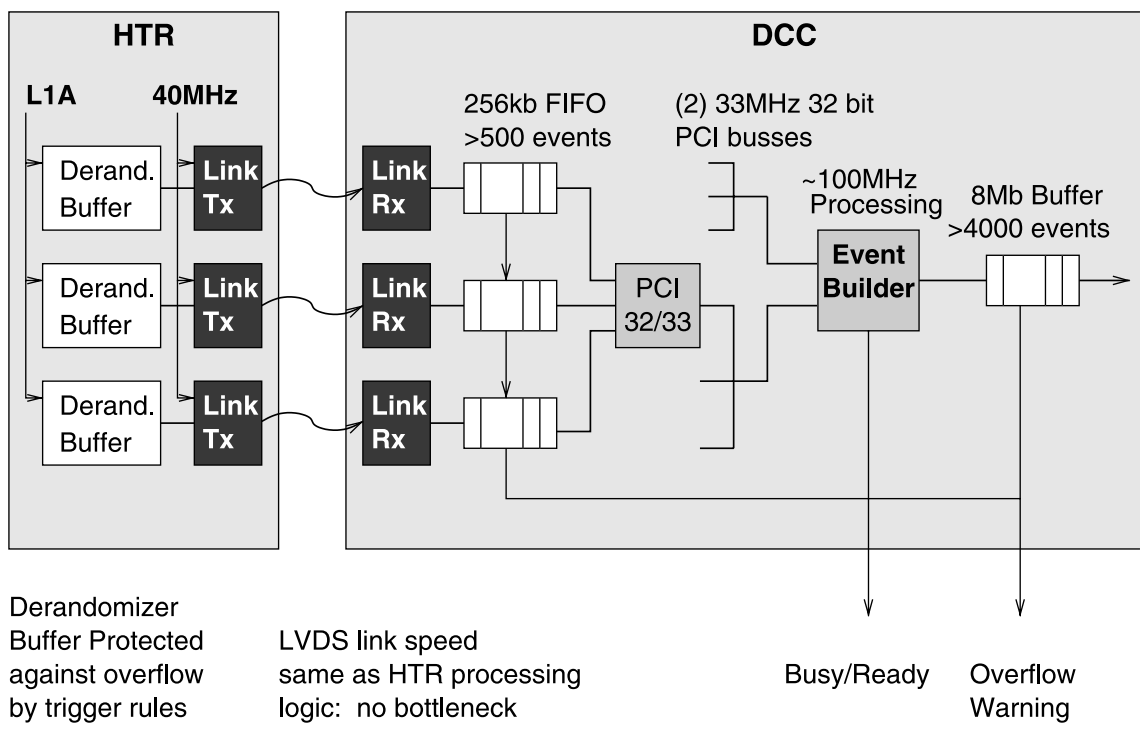

Fig. 7. HCAL DAQ buffering schematic. The HTR sends the received data to the DCC. The DCC stores event fragments in FIFO buffers on the input and output 
All single-bit errors are corrected and all double-bit errors are detected by this technique. Event synchronization is checked by means of an event number in the header and trailer of each event, which are checked against the TTC event number.

\section{Test beam setup and preliminary measurements}

The data were recorded during 2002 at the CERN H2 test beam. A moving platform held two production HB wedges plus a pre-prototype electromagnetic crystal calorimeter which was inserted in front of the HB for some of the measurements. The two-dimensional movement of the platform in $\phi$ and $\eta$ directions allowed the beam to be directed onto any tower of the calorimeter. Four scintillation counters were located approximately three meters upstream of the calorimeters and a coincidence between a subset of these counters was used for the trigger. The beam size varied depending on the beam energy and magnet settings but was typically several millimeters in the transverse dimensions.

A pre-prototype electromagnetic calorimeter module was constructed from 49 lead tungstate crystals arranged in a $7 \times 7$ array and coupled to individual photomultiplier tubes. The front face dimensions are approximately $2.2 \times$ $2.2 \mathrm{~cm}^{2}$, and the length of the crystal is $23 \mathrm{~cm}$ or 25.8 radiation lengths. From subsequent test beam data it became clear that production ECAL modules are far superior to this pre-production module. Even though data were taken with this ECAL module, the results will not be discussed further here but in a subsequent paper where EB production modules were available.

\subsection{Data sets}

Data were taken with electron beam momenta of 20,30 , 50 and $100 \mathrm{GeV} / c$, pion beam momenta of 20,30, 50, 100 , and $300 \mathrm{GeV} / c$, and a muon beam of momentum $225 \mathrm{GeV} / c$. The momentum spread of the pion beam was established by collimators which were typically set to provide momentum spread that is less than $\Delta p / p= \pm 1 \%$, and the beam momentum spread contributes negligibly to the measured calorimeter resolution. The test beam is created by extracting the primary proton beam over $1.2 \mathrm{~s}$ and impinging on a primary target in the SPS in CERN. The beam is spread uniformly with no particular time structure. The time of each beam particle is determined by a TDC to an accuracy of about $1 \mathrm{~ns}$ using the beam counters.

Beams of muons, pions and electrons were directed into the centers of all accesible towers, which included all four $5^{\circ}$ sectors of the bottom wedge, but was limited to the bottom two $5^{\circ}$ sectors of the top wedge for mechanical reasons. For both wedges, the scan covered the entire $\eta$ range (16 sectors). These data were taken without the electromagnetic calorimeter module.
Additionally, several special runs were taken with the moving radioactive wire source which illuminated 2092 tiles for the two wedges and with a LED pulser which injected short light pulses to the HPDs.

\subsection{Laser calibration and monitoring system}

In addition to a LED light injection system, a $300 \mathrm{~mJ}$ nitrogen laser is used to monitor the calorimeter and check on the long term relative time calibration. The laser light at $337 \mathrm{~nm}$ has two paths. The first path carries the UV light by quartz fibers to a tiny green scintillator inside the RBX housing and lights up all 19 pixels of each HPD. The second path carries the UV light with another quartz fiber path to layer 9 scintillator of each tower. The quartz fiber path length is adjusted so that the timing of the signals in each tower mimics (within $1 \mathrm{~ns}$ ) an actual event in the center of the CMS detector. The laser system was tested in the test beam and indeed the timing accuracy was within one nanosecond.

\subsection{Noise performance}

The front-end electronics was operated at $33.79 \mathrm{MHz}$ for all measurements reported in this paper. Figure 8 shows the noise distribution at the highest gain setting of the multirange ADC for a single QIE channel. The signal is summed over four time slices, and the signal arrives within $2 \mathrm{~ns}$ at the start of the second time slice. The least count corresponds to 2080 electrons measured by precise charge injection. The observed noise spread in the pedestal is about 5000 electrons. From the beam measurements, this corresponds to an energy of $\sim 0.2 \mathrm{GeV}$. After the 2002 test beam, the front-end electronics was upgraded to $40 \mathrm{MHz}$, the operational mode of the LHC. There is no significant common mode noise.

\subsection{Time structure}

The time structure of the HB pulse is dominated by the decay time of the wavelength shifting fibers. The front-end electronics, including the photo-detector is fast enough not to distort the pulse shape. An accurate knowledge of the pulse shape is essential to correlate energy deposit with a particular LHC beam crossing. We made two independent measurements of the time structure of the pulse of deposited energy. First, we investigated with a photomultiplier and commercial electronics; and second, we used the HPD and QIE electronics.

\subsubsection{Measurements with a photomultiplier tube}

We used a single 10-stage, 2-inch photo-multiplier tube (RCA 6342A) and the output was fed into a digital oscilloscope which recorded voltage in $0.4 \mathrm{~ns}$ bins. For this study, the output of $3 \times 3$ towers were read by the photomultiplier tube, with the beam aimed at the center of 

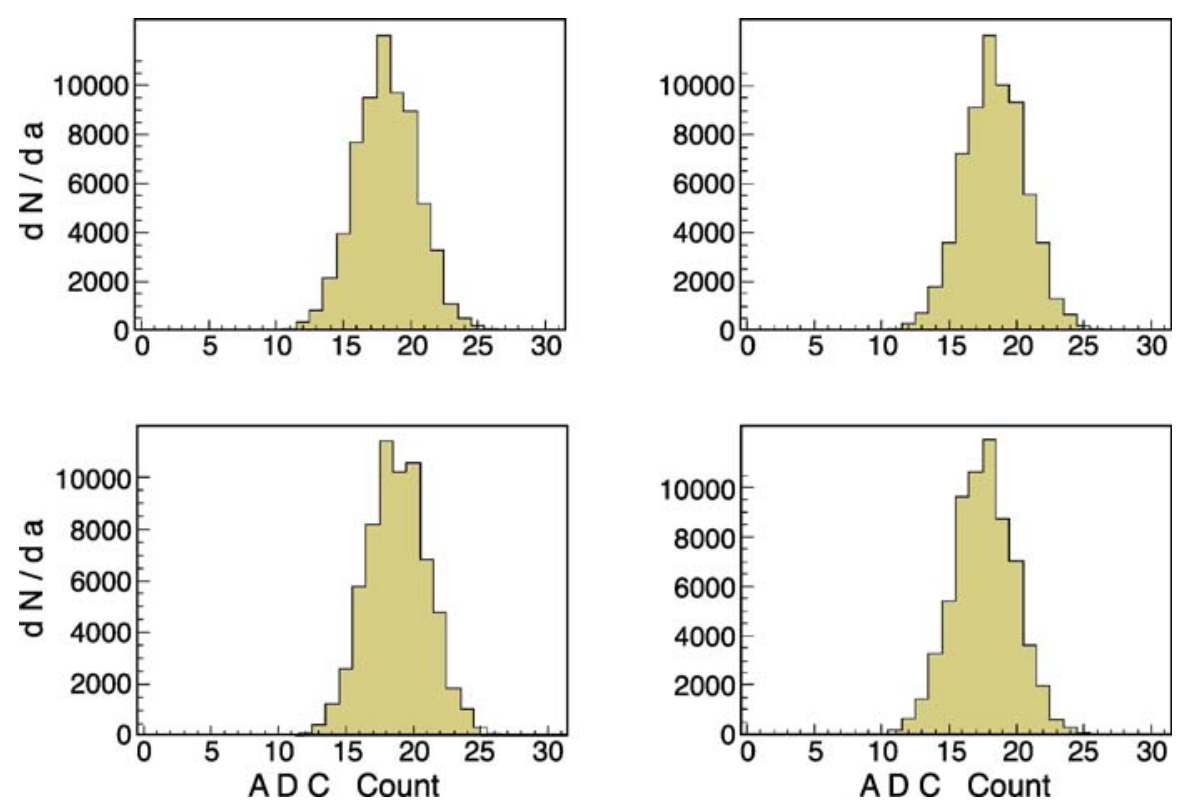

Fig. 8. Measured pedestal distributions $(\mathrm{d} N / \mathrm{d} a)$ in units of raw ADC counts in the most sensitive QIE range. The rms is 2.3 ADC counts
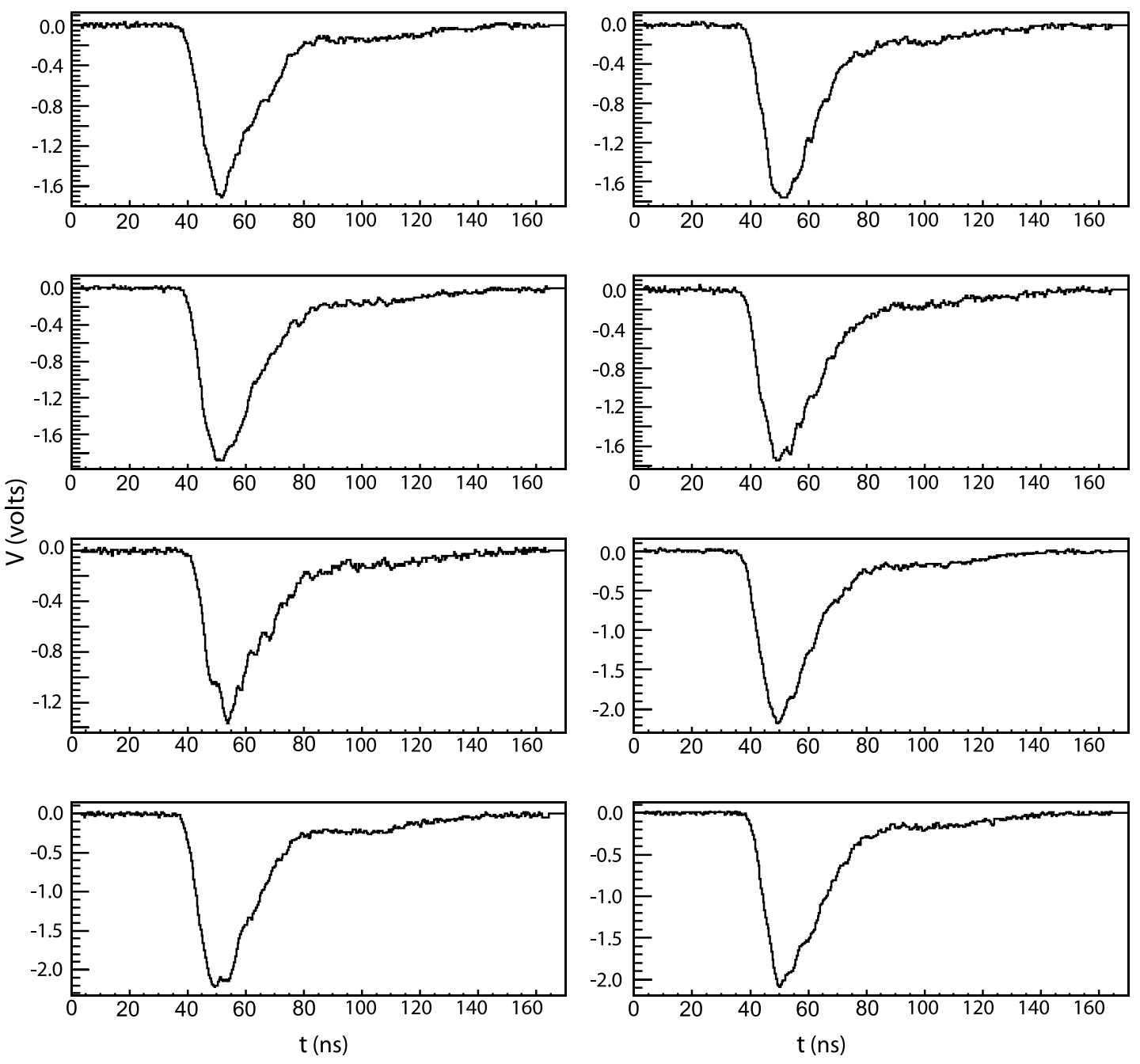

Fig. 9. Calorimeter pulses for eight individual events, voltage vs. time, observed with a photomultiplier for $300 \mathrm{GeV} / c$ pion showers 

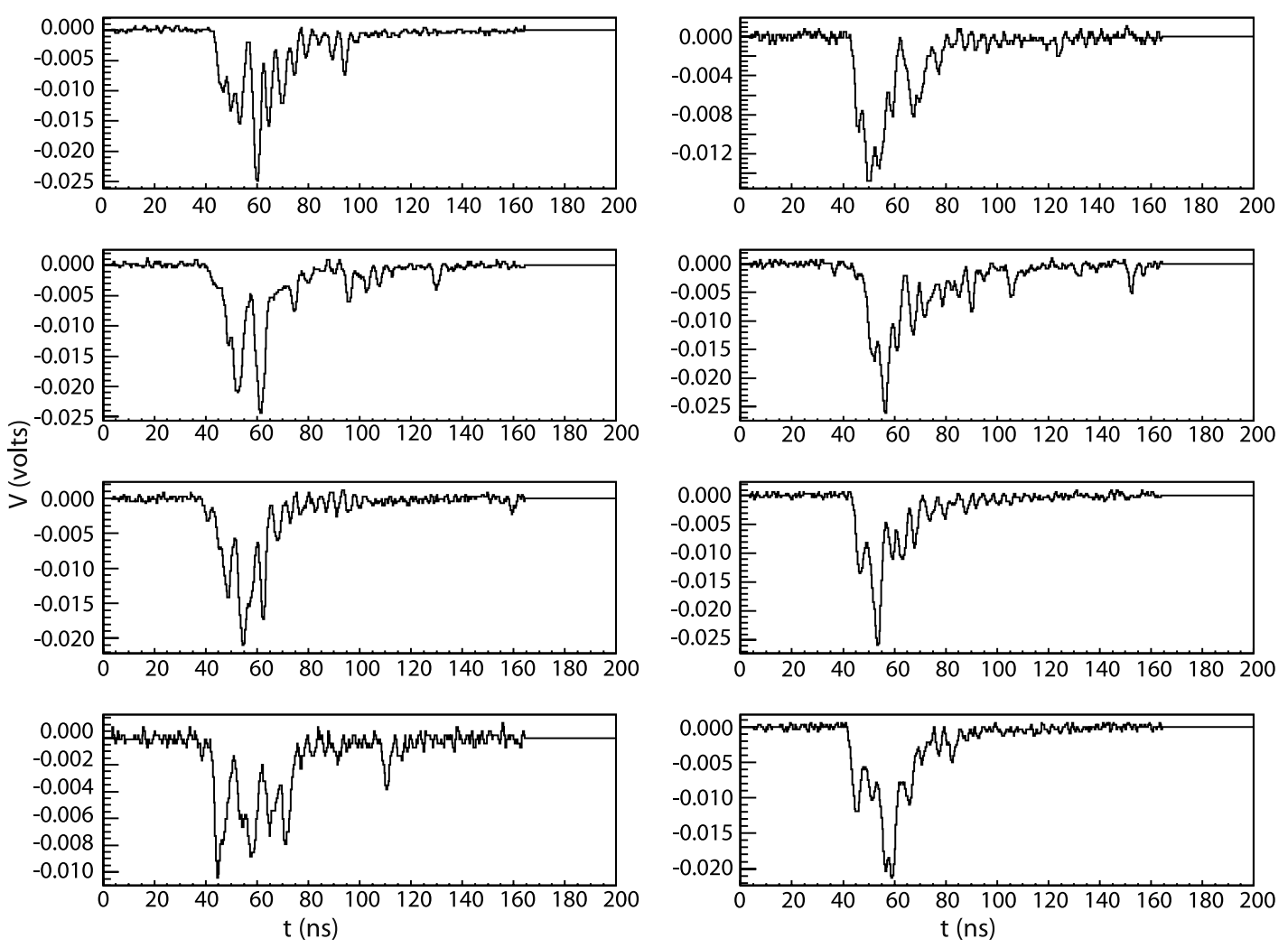

Fig. 10. Calorimeter pulses for eight individual events, voltage vs. time, observed with a photomultiplier for $225 \mathrm{GeV} / c$ incident muons
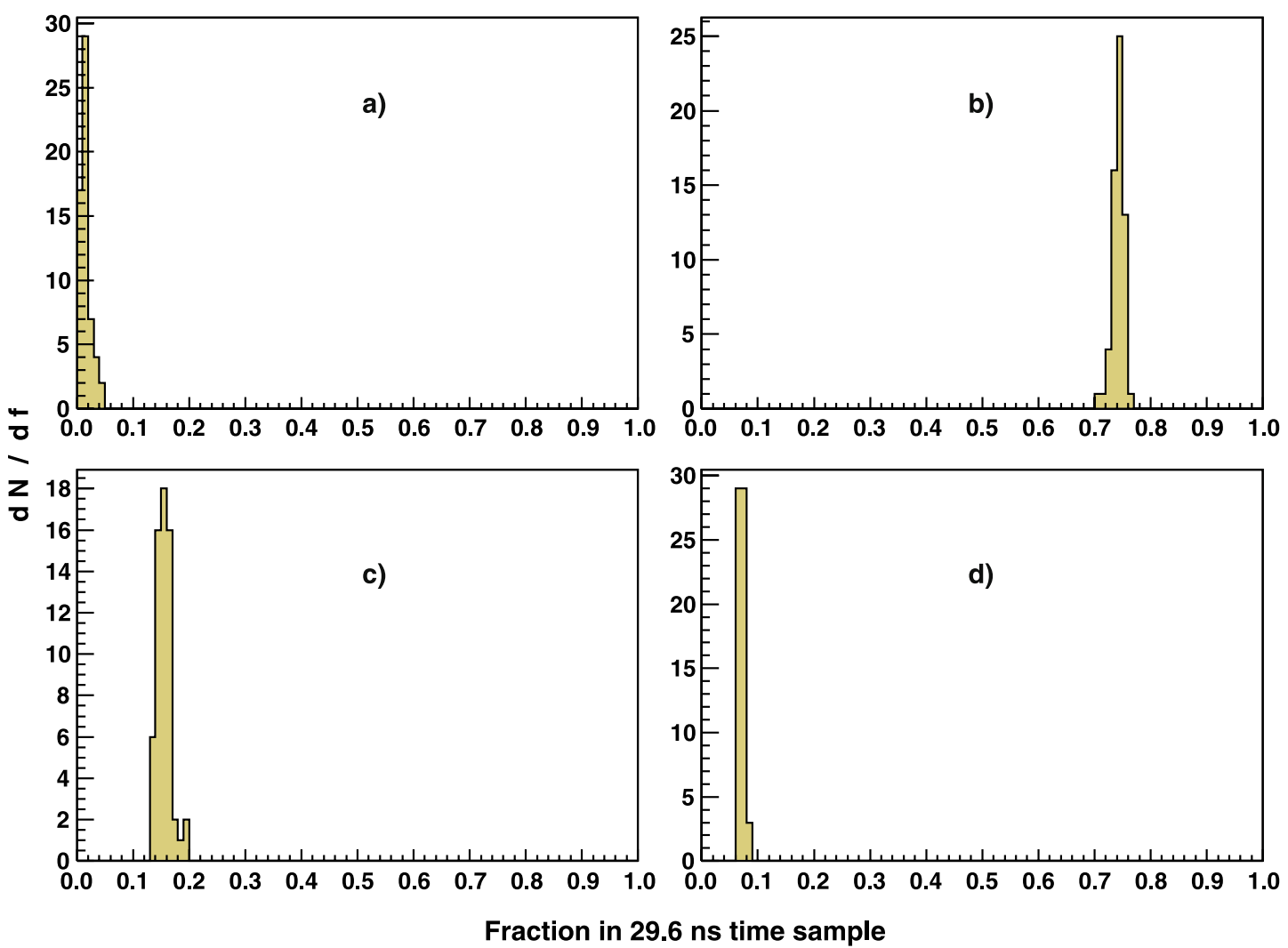

Fig. 11. Fraction of energy $(f)$ observed in $29.6 \mathrm{~ns}$ time samples for $200 \mathrm{GeV} / c$ pion showers. a First time sample, b second time sample, c third time sample, and $\mathbf{d}$ fourth time sample

the central tower. We recorded 300 and $20 \mathrm{GeV} / c$ pion and $100 \mathrm{GeV} / c$ electron showers, as well as signals from high energy muons. Data from $300 \mathrm{GeV} / c$ pions and $225 \mathrm{GeV} / c$ muons are shown in Figures 9 and 10, respectively. In the case of muons, fluctuations in the number of photoelectrons lead to large event by event fluctuations. 


\subsubsection{HPD/QIE measurements}

Twenty time samples were recorded for each QIE channel per event. The timing was adjusted so that the event occurred near the middle of the time sequence (sample number 10) allowing us to record the noise performance well before and after the energy pulse. At a frequency of $33.79 \mathrm{MHz}$, each time sample corresponds to $29.6 \mathrm{~ns}$ (slightly longer than the $25 \mathrm{~ns}$ between LHC bunch crossings). Figure 11 shows the fraction of energy observed in four consecutive time bins of width $29.6 \mathrm{~ns}$. The energy was summed over $3 \times 3$ towers, with the beam positioned in the center of the central tower. Using the TDC time information from the beam counters, the events were selected where the beam signal starts in the first $2 \mathrm{~ns}$ of the second of four time slices. Most of the signal is collected in two time samples. Note that $\sim 75 \%$ of the total energy arrives in a single time sample and this fraction has small dispersion. The reduced clock frequency used in these tests is the result of a design problem in the first procured QIE chips used at the time of data taking. Subsequent beam tests with the upgraded electronics functioning at $40 \mathrm{MHz}$ showed essentially the same results.

Figure 12 shows pulse shapes for $30 \mathrm{GeV} / c$ electrons and $300 \mathrm{GeV} / c$ pions. For this measurement the signal from $3 \times 3$ towers around the beam was read by the QIEs. The timing information was obtained by using the TDC information provided by the beam counters. The time distribution of the signal is completely dominated by the wavelength shifting green fibers. The pulse shape was stable for showers for momentum range from 20 to $300 \mathrm{GeV} / c$ and was independent of particle type.

If we select the phase of the test beam particles by requiring that $10 \%-12 \%$ of the observed energy is in the 10th time sample, then all the events have the same temporal shape. We have the freedom to adjust this phase during CMS running using programmable delays provided for each individual channel of the front-

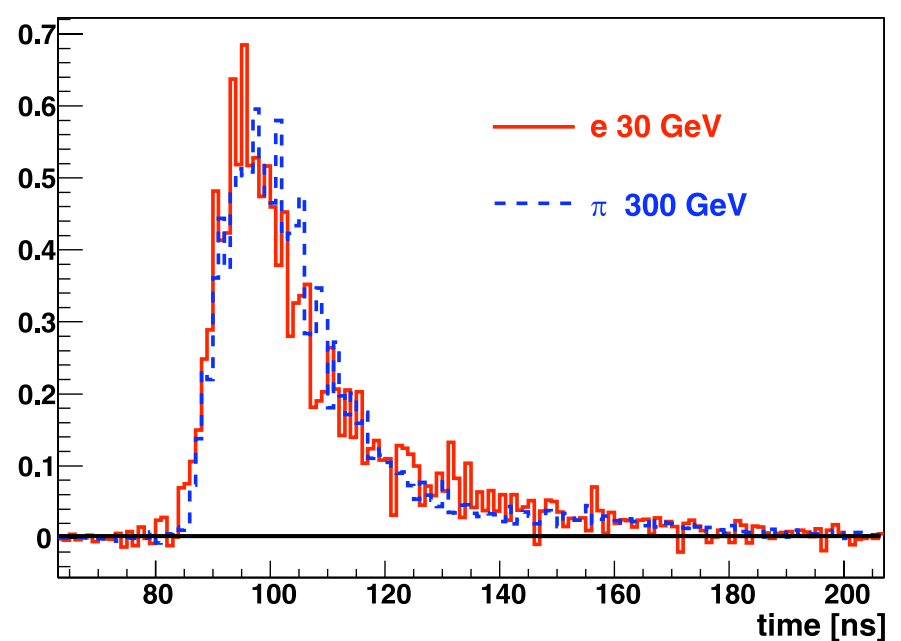

Fig. 12. Pulse shape for $30 \mathrm{GeV} / c$ electrons and $300 \mathrm{GeV} / c$ pions

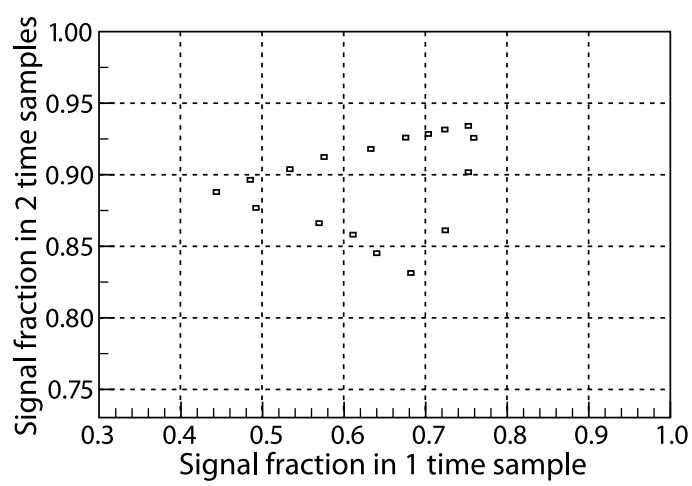

Fig. 13. Fraction of signal observed in two time samples vs. in a single time sample as the phase is changed in 18 intervals spanning a complete time sample. The zero point is suppressed for each axis

end electronics. Figure 13 shows the fraction of energy in two time samples (vertical scale) versus the fraction of energy observed in a single time sample (horizontal scale). The data points represent different choices of the phase. It is possible to have $75 \%$ of the energy in a single time sample and more than $90 \%$ of the energy in two time samples. Note that the signal fraction measurement thus can provide an arrival measurement of $\sim 2 \mathrm{~ns}$.

The QIE introduces a time delay of up to the order of $10 \mathrm{~ns}$ depending on pulse height. This is due to the input impedance of the QIE. The time slew can be reduced at the expense of increasing the amount of noise. Figure 14 shows the time slew effect measured in a test setup in the laboratory and test beam measurements. The production modules were constructed with about $5000 e$ noise and a maximum time slew for very low energy particles of up to $10 \mathrm{~ns}$. Pulse height measurements can be used to correct for this effect.

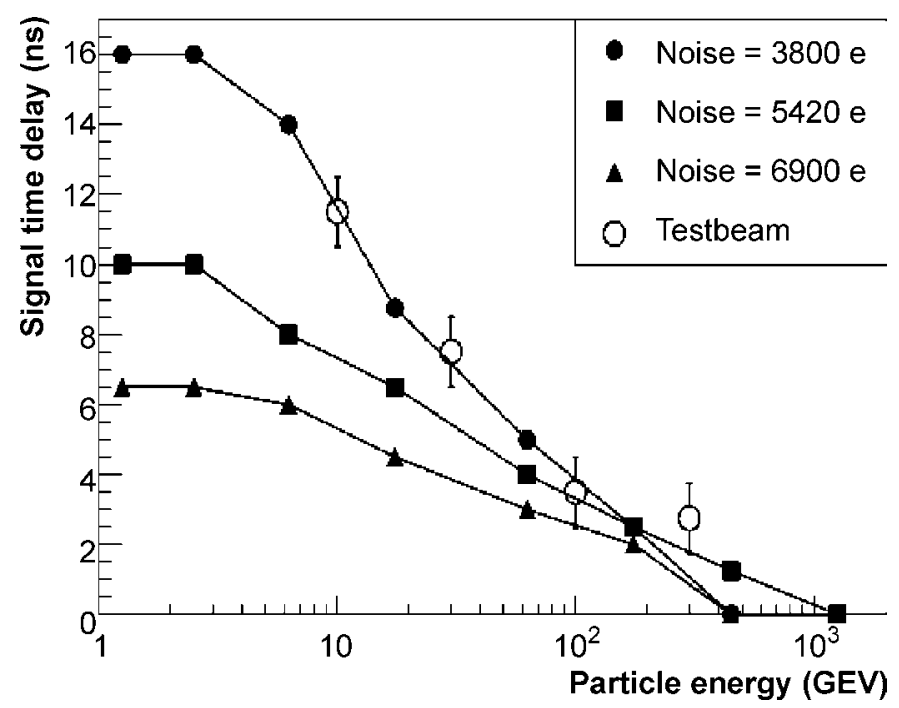

Fig. 14. Time slewing vs. energy for laboratory bench and test beam measurements for three choices of pedestal noise 


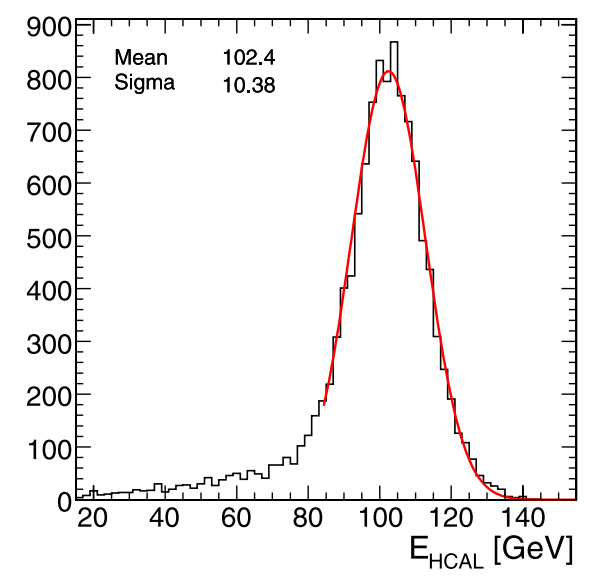

Fig. 15. The energy distribution in the HB $(5 \times 5$ towers $)$ for $100 \mathrm{GeV} / c$ pions. The solid line is a single Gaussian fit to the histogram data with best fit values for the Gaussian parameters shown in the figure legend

\section{The HB response to pions and muons}

The absolute energy scale of the HB was defined using a beam of $50 \mathrm{GeV} / c$ pions directed at the calorimeter. Even though the pre-prototype EB was in the beam, only those pions which deposited less than $2 \mathrm{GeV}$ in the EB were selected for the HB calibration. The calibration factor was extracted as the ratio between the pion beam momentum and the mean of the distribution of summed HB charge deposited in a $5 \times 5$ tower array centered on the beam position. Typical calibration constant is $0.2 \mathrm{GeV} / \mathrm{fC}$.

With the $100 \mathrm{GeV} / c$ pion beam centered on a tower, the fraction of energy observed in the central tower is on average $82 \%$. If the energy in the neighboring eight towers is added, forming a $3 \times 3$ array, $98 \%$ of the pion energy is observed. The energy response for $100 \mathrm{GeV} / c$ pions in a $5 \times 5$ array is shown in Fig. 15. The beam was centered at tower 4 in $\eta$ direction, and the energy was summed over four time slices. As stated earlier, only events that deposited less than $2 \mathrm{GeV}$ in the electromagnetic calorimeter were selected, and most of these events deposited less than $1 \mathrm{GeV}$ in the EB. The figure shows the energy in the HB alone. A Gaussian fit gives the energy resolution $\sigma$ at this energy of about $10 \%$. The low energy tail in Fig. 15 is due to leakage of late developing showers. The outer hadron (HO) calorimeter in CMS is designed to capture this leakage but it was not installed during these tests.

It is important to understand the response of the HB to muons in order to provide redundant calibration to the primary radioactive source method but also for particle identification purposes. A feature bit will be reported for use in higher level triggers, when a muon is identified by the HB. The signal, with $225 \mathrm{GeV} / c$ incident muons, is about $2.5 \mathrm{GeV}$ on average and clearly visible above the electronic noise $(\sim 0.2 \mathrm{GeV})$. Figure 16 shows the muon energy response from a single tower. A Landau fit to this distribution results in $1.64 \mathrm{GeV}$ for the most probable and $2.5 \mathrm{GeV}$ for the mean value. A hadronic shower deposits most of its energy in two or three depth layers with large event

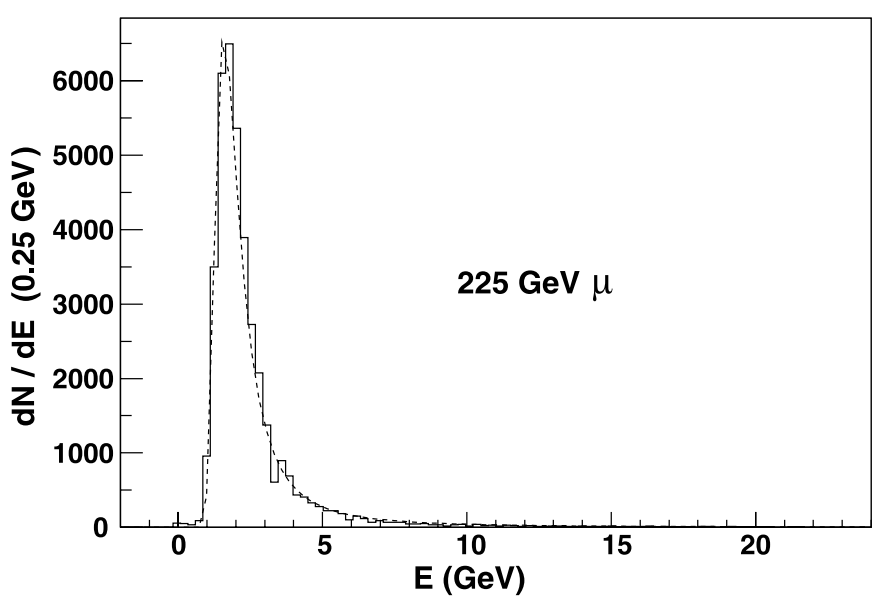

Fig. 16. Observed energy distribution in the $\mathrm{HB}$ for $225 \mathrm{GeV} / c$ incident muons. The most probable value for the energy deposited is $1.64 \mathrm{GeV}$ while the mean is $2.5 \mathrm{GeV}$. The dashed curve is the result of a fit by a Landau distribution

by event fluctuations, so the constant term is substantially larger.

\section{Radioactive source calibration}

The radioactive source injects the same amount of energy into each tile of each tower. The DC value of the source current is measured by the QIEs at the center of each tile by reading the total charge every $25 \mathrm{~ns}$. The rms of the pedestals is about $0.7 \mathrm{fC}$. The signal above pedestal is about $1 \mathrm{fC}$, depending on the strength of the source with an rms value of about $1 \mathrm{fC}$. During a typical measurement of a tile about 250000 measurements are recorded, and the rms of the average signal above pedestal is about $1 \mathrm{fC} / \sqrt{250000}=0.002 \mathrm{fC}$. Even though an individual recording of a measurement has a error of about $100 \%$, the very large number of measurements reduces the error of the mean to $0.2 \%$. The normalization is performed by comparing the sum of the tile currents (suitably weighted with an average shower shape in depth) to the energy response of the tower in the test beam of known energy. The achieved absolute calibration is better than $2 \%$ and reproducible to $1 \%$ [16].

The radioactive source calibration by ${ }^{137} \mathrm{Cs}\left(\right.$ or ${ }^{60} \mathrm{Co}$ ) is performed for every scintillator tile. The ratio of the radioactive source signal to the energy response to pions in the beam for each tower provides the initial calibration. This ratio depends on the type and activity of the source. The signal means from 1745 tiles, corrected for the different fiber attenuation, are shown as a histogram in Fig. 17. The rms width of the distribution is $8 \%$ and it is consistent with the measurements of tile uniformity made during tray construction.

The dependence of the ratio of radioactive signal to the $100 \mathrm{GeV} / c$ electron beam signal on the tower number is shown in Fig. 18. The same dependence for the ratio of radioactive signal to the muon signal is presented in Fig. 19. 


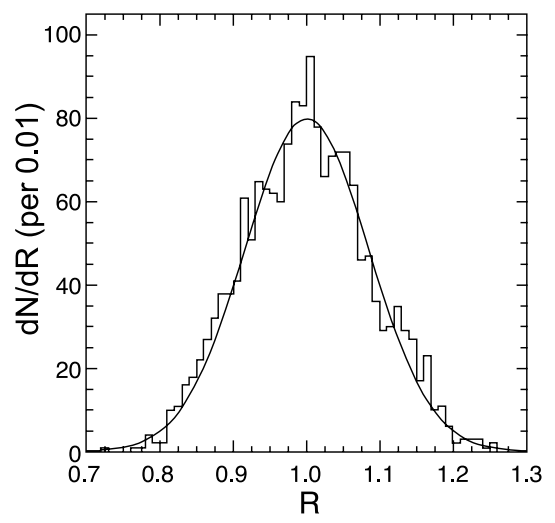

Fig. 17. Distribution of scintillating tile response $(R)$ to the source calibration normalized to the mean of the distribution. The line represents a single Gaussian fit to the data. The fitted rms spread of about $8 \%$ is consistent with the spread in values measured with the collimated source made during tile assembly

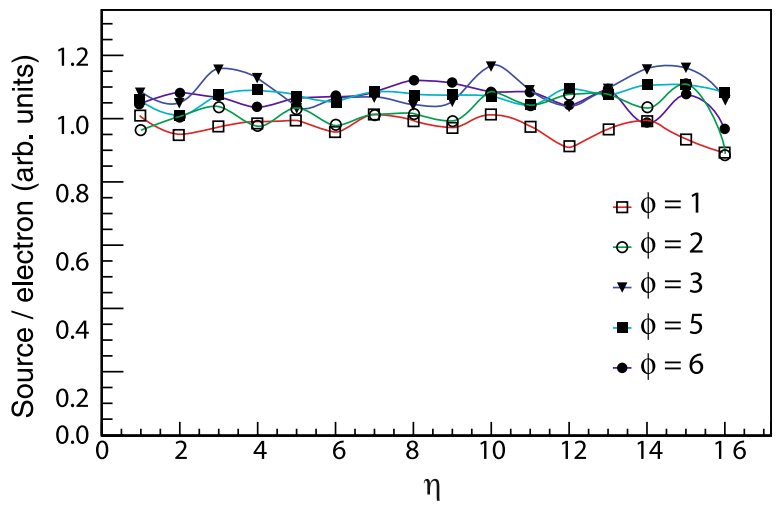

Fig. 18. Ratio of the radioactive source signal (fit to peak value) to the $100 \mathrm{GeV} / c$ incident electron signal in the HB vs. $\eta$ number of the tower for five different $\phi$ numbers (80 towers total)

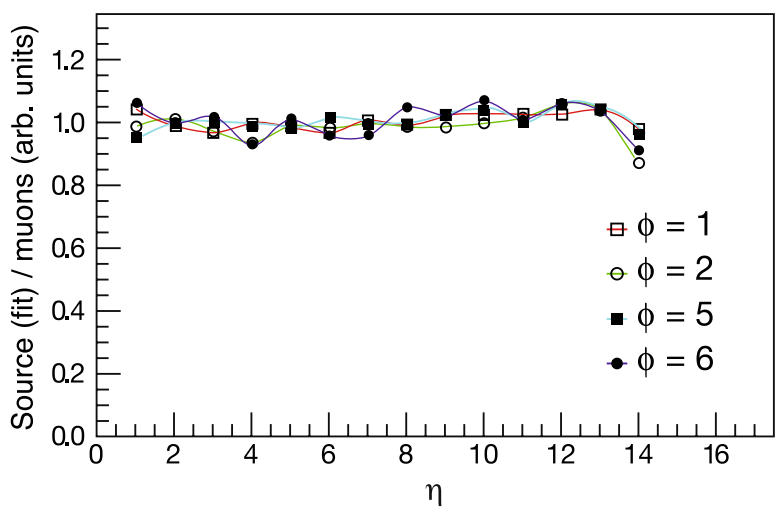

Fig. 19. Ratio of the radioactive source signal to the HB signal due to a $225 \mathrm{GeV} / c$ beam of incident muons vs. $\eta$ number for four different $\phi$ numbers (64 towers total)

The electron response agrees with the radioactive source measurements to an rms of $5 \%$, while the muon data agree to $3 \%$. The greater spread in the electron data is due to

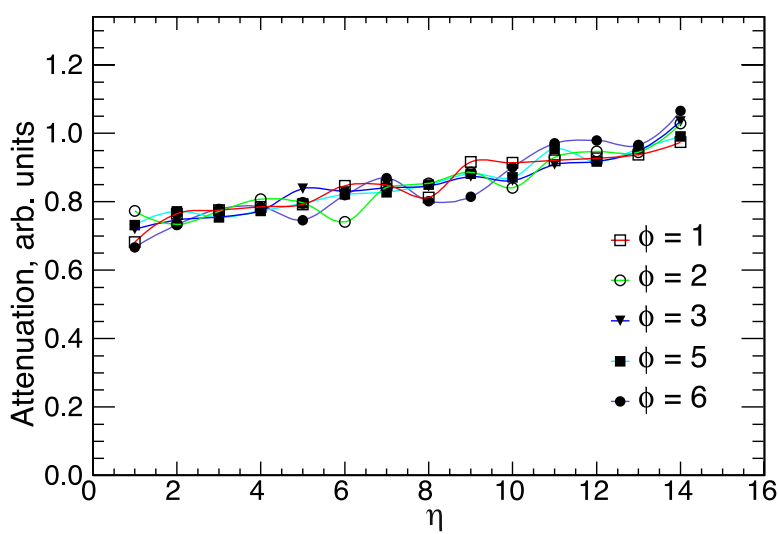

Fig. 20. Measurement of the attenuation vs. $\eta$ number for five different $\phi$ numbers (80 towers total). The attenuation occurs in the propagation of the signals in fibers from the towers to the readout box which is located on the $\eta=14$ end of the wedge

the fact that the electron shower is concentrated in the first few scintillator layers, while the source measurement is averaged over all layers with equal weight as is the muon signal. While the muon data are better suited to establish the HB tower-to-tower relative calibration, the data from electrons establish the absolute energy scale. Therefore, we have a cross check of the calibration established by pions.

The length of the optical fibers between the scintillators and the HPDs varies with $\eta$. Figure 20 shows the relative response to the radioactive source as a function of $\eta$. These data show that the light level at the HPD is about $30 \%$ lower for $\eta=1$ towers when compared to $\eta=14$ towers. The $\eta=15$ and 16 towers are not included in these measurements because they are segmented longitudinally.

\section{Summary and conclusions}

We have described the design and construction of the CMS HCAL barrel calorimeter. The results presented in this paper come from the first data set with the nearly final electronics that was available at the time of the beam tests (2002). The HB electronics noise is $\mathrm{HB} 0.2 \mathrm{GeV}$ per tower. The pulse shape was determined to be $75 \%$ contained in a single 29.6 ns time sample. A radioactive source system was used to record the response of each scintillator tile of HB to the source. The relationship of the HB response to particle beams and sources then established a method to extend the calibration to all those HB modules which were not exposed to particle beams. Thus, these data are used to establish the initial (pre-LHC beam) calibration of the entire HB detector. Since only four HB wedges out of 36 wedges were eventually calibrated in the test beam, it is crucial that the measurements by the radioactive wire source to be used for calibration give the correct energy calibration for all the wedges. The measurements described in this paper show that the consistency between the test beam and the wire source measurements is better than $4 \%$ for hadron showers. Thus, the wire source measurements 
of every tile of every tower of every wedge will have a calibration constant (initial) to about $4 \%$. The calibration will be improved in the CMS by physics events. The Hadron Barrel calorimeter design and construction meets the requirements for physics as stated in the Technical Design Report and will be ready to take data on day one of LHC operations.

Acknowledgements. This project was carried out with financial support from CERN, the U.S. Department of Energy (DoE), the U.S. National Science Foundation (NSF), RMKI-KFKI (Hungary, OTKA grant T 016823), the Scientific and Technical Research Council of Turkey (TÜBITAK), Turkish Atomic Energy Agency (TAEK), Bogazici University Research Fund (Grant no: 04B301), the Russian Ministry for Education and Science and the Russian State Committee for Atomic Energy.

\section{References}

1. CMS Technical Proposal, CERN/LHCC 94-38, LHCC/P1 (December 1994)

2. The LHC design report and current status is available at http://lhc-new-homepage.web.cern.ch/lhc-newhomepage/

3. The Hadron Calorimeter Technical Design Report, CERN/ LHCC 97-31, CMS TDR 2 (June 1997)

4. The TriDAS Project, Technical Design Report, Volume 1: The Trigger Systems, CERN/LHCC 2000-38, CMS TDR 6.1 (December 2000)

5. The TriDAS Project, Technical Design Report, Volume 2: Data Acquisition and High-Level Trigger, CERN/LHCC, CMS TDR 6.2 (December 2002)

6. Physics Technical Design Report, Volume 1, CERN/LHCC 2006-001 (February 2006)
7. V.I. Kryshkin, A.I. Ronzhin, Nucl. Instrum. Methods A 247, 583 (1986)

8. M.G. Albrow et al., Nucl. Instrum. Methods A 256, 23 (1987)

9. G.W. Foster, J. Freeman, R. Hagstrom, Nucl. Phys. B 23, 93 (1991)

10. P. de Barbaro et al., Nucl. Instrum. Methods A 315, 317 (1992)

11. CMS-HCAL Collaboration, G. Baiatian et al., Design, Performance, and Calibration of CMS Forward Calorimeter Wedges, CMS NOTE-2006/044

12. CMS-HCAL Collaboration, G. Baiatian et al., Design, Performance, and Calibration of CMS Hadron-Barrel Calorimeter Wedges, CMS NOTE-2006/138

13. CMS-HCAL Collaboration, G. Baiatian et al., Synchronization and Timing in the CMS Hadron Calorimeter, CMS NOTE-2006/139

14. CMS-HCAL Collaboration, G. Baiatian et al., Energy Response and Longitudinal Shower Profiles Measured in CMS HCAL and Comparison with GEANT4, CMS NOTE2006/143

15. V.V. Abramov et al., Nucl. Instrum. Methods A 457, 75 (2001)

16. E. Hazen et al., Nucl. Instrum. Methods A 511, 311 (2003)

17. The Electromagnetic Calorimeter Technical Design Report, CERN/LHCC 97-33, CMS TDR 4 (December 1997)

18. E. Hernandez et al., Radiat. Phys. Chem. 41, 409 (1993)

19. P. Cushman, A. Heering, A. Ronzhin, Nucl. Instrum. Methods A 442, 289 (2000)

20. A. Bream et al., Nucl. Instrum. Methods A 478, 400 (2002)

21. T. Zimmerman, M. Sarraj, IEEE Trans. Nucl. Sci. 43, 1683 (1996)

22. T. Zimmerman, J.R. Hoff, IEEE J. Solid State Circuits 39, 895 (2004)

23. The Channel Link is described in the National Semiconductor data sheet, DS90CR285/DS90CR286, +3.3V Rising Edge Data Strobe LVDS 28-Bit Channel Link-66 MHz, (November 2000) 